Supporting Information

\title{
A 3d-4f Metal-Organic Framework with Dual Luminescent Centers Efficiently Discriminates the Isomer and Homologues of Small Organic Molecules
}

Guang Zeng, Shanghua Xing, Xiuru Wang, Yulin Yang, Dingxuan Ma, Hongwei Liang, Lu Gao, Jia Hua, Guanghua Li, * Zhan Shi, and Shouhua Feng

State Key Laboratory of Inorganic Synthesis and Preparative Chemistry, College of Chemistry, Jilin University, Changchun 130012 (P. R. China) 


\section{Materials and Characterization.}

All reagents and sol-vents used in the syntheses were obtained from commercial sources without any purification. Element alanalyses were measured on a Perkin-Elmer 2400 elemental analyzer. UV/Vis spectra were recorded on a UV-2450 UV-Visible spectrophotometer within the wavelength range $200-500 \mathrm{~nm}$ using the same solvent in the examined solution as a blank. The infrared (IR) spectra were recorded within the $400-4000 \mathrm{~cm}^{-1}$ regionon a Nicolet Impact 410 FTIR spectrometer with $\mathrm{KBr}$ pellets. Thermogravimetric experiments were carried out by a TGA Q500 V20.10 Build 36 from room temperature to $800{ }^{\circ} \mathrm{C}$ at a heating rate of $10{ }^{\circ} \mathrm{C} \mathrm{min}{ }^{-1}$. The X-ray powder diffraction (XRPD) experiments were performed in a Rigaku D/max 2550 X-ray powder diffractometer with $\mathrm{Cu} \mathrm{K} \alpha$ radiation. The quantum yields were measured with an integrating sphere (C-701, Labsphere Inc.), a $365 \mathrm{~nm}$ Ocean Optics LLS-LED as the excitation source and the laser was introduced into the sphere through the optical fiber. The luminescence spectra were measured on a FluoroMax-4 fluorescence spectrometer at room temperature.

\section{The calculation of quantum yield.}

The fluorescence Quantum Yield (QY) is the probability of a sample emitting a photon through fluorescence when it is excited by a single photon. It is therefore also the percentage of photons emitted by a bulk sample when a given number of photons are absorbed by the sample, i.e. the number of photons emitted divided by the number of photons absorbed by a sample. The number of photons absorbed by a bulk sample is equal to the number of photons incident on the sample minus the photons passing through and not being absorbed by it.

The quantum yield can thus be represented simply in the equation below. If the following 
three parameters are known: 1 , number of photons incident on the sample volume; 2 , number of photons not absorbed by the sample; 3 , number of photons emitted by the sample.

$$
\eta_{1}=\frac{\varepsilon}{\alpha}=\frac{\int L_{\text {emission }}}{\int E_{\text {solvent }}-\int E_{\text {sample }}}
$$

Where $\boldsymbol{\varepsilon}$ is the photons emitted by the sample

$\eta$ is the quantum yield

Lemission is the luminescence emission spectrum of the sample, collected using the sphere Esample is the spectrum of the light used to excite the sample, collected using the sphere Esolvent is the spectrum of the light used for excitation with only the solvent in the sphere, collected using the sphere.

The parameters can be represented by the convention used in Figure 8 as:

$$
\eta_{1}=\frac{\int L_{\text {direct }}}{\int E_{\text {without }}-\int E_{\text {direct }}}
$$

When it is possible, a series of indirect measurements should also be made to measure and remove the re-excitation of the sample from excitation light reflected within the sphere. Thus a second equation for QY is considered more accurate.

$$
\eta_{2}=\frac{\left(E_{\text {indirect }} L_{\text {direct }}\right)-\left(E_{\text {direct }} L_{\text {indirect }}\right)}{\left(E_{\text {indirect }} E_{\text {without }}\right)-\left(E_{\text {direct }} E_{\text {without }}\right)}-\text { Ref. 1 }
$$

Ref. 1: 'An Improved Experimental Determination of External Photoluminescence Quantum Efficiency’ Advanced Materials. 1997, 9, No 3, page 230 
Synthesis of 3-(pyridine-4-yl) benzoic acid (Hpba)

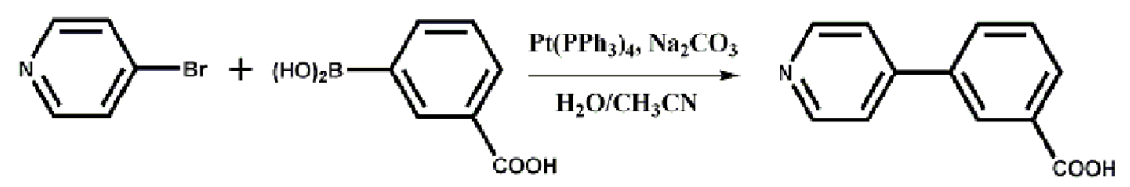

Ligand (Hpba) was synthesized according the previous report. ${ }^{2}$ To a degassed solution of 3-Carboxyphenylboronic acid (1.70 g, $10.2 \mathrm{mmol})$, 4-bromopridine hydrochloride (2.01 g, 10.3 mmol), and sodium carbonate $(2.10 \mathrm{~g}, 19.8 \mathrm{mmol})$ in $100 \mathrm{~mL}$ of a mixed solution $(1: 1$ $\left.\mathrm{CH}_{3} \mathrm{CN}: \mathrm{H}_{2} \mathrm{O}\right)$ at room temperature, $\mathrm{Pd}\left(\mathrm{PPh}_{3}\right)_{4}(0.30 \mathrm{~g}, 0.26 \mathrm{mmol})$ was added under $\mathrm{N}_{2}$ pressure. The reaction mixture was refluxed for $22 \mathrm{~h}$. the hot suspension was filtered. The filtrate was concentrated to about half of the original volume and then washed with $\mathrm{CH}_{2} \mathrm{Cl}_{2}$. The aqueous layer was acidified with concentrated $\mathrm{HCl}$; the resulting white precipitate was collected, washed with $\mathrm{CH}_{3} \mathrm{CN}$.

2. Sekiya, R.; Nishikiori, S.; Ogura, K. Inorganic Chemistry, 2006, 45, 9233.

Crystal X-ray crystallography. Single crystal diffraction data for compound $\mathbf{4}$ and the analogues were collected on a Rigaku RAXIS-RAPID equipped with a narrow-focus, $5.4 \mathrm{~kW}$ sealed tube X-ray source (graphite-monochromated Mo K $\alpha$ radiation, $\lambda=0.71073 \AA$ ). Data processing was accomplished with the PROCESS-AUTO processing program. Direct methods were used to solve the structure using the SHELXL crystallographic software package. All non-hydrogen atoms were easily found from the difference Fourier map. All non-hydrogen atoms were refined anisotropically. The crystallographic information and structure refinement details are summarized in Table S1 
Table S1. Crystal Data Collection and Structure Refinement for Compound 1, 2, 3 and 4.

\begin{tabular}{|c|c|c|c|c|}
\hline Compound & Compound 1 & Compound 2 & Compound $\mathbf{3}$ & Compound 4 \\
\hline $\begin{array}{l}\text { Temperatu } \\
\text { re/K }\end{array}$ & $298 \mathrm{~K}$ & $298 \mathrm{~K}$ & $298 \mathrm{~K}$ & $298 \mathrm{~K}$ \\
\hline Formula & $\begin{array}{c}\mathrm{LaCu}_{4} \mathrm{I}_{4} \mathrm{C}_{46} \mathrm{H}_{47} \mathrm{~N}_{3} \\
\mathrm{O}_{13}\end{array}$ & $\begin{array}{c}\mathrm{CeCu}_{4} \mathrm{I}_{4} \mathrm{C}_{46} \mathrm{H}_{48} \mathrm{~N}_{3} \\
\mathrm{O}_{13}\end{array}$ & $\begin{array}{c}\mathrm{EuCu}_{4} \mathrm{I}_{4} \mathrm{C}_{46} \mathrm{H}_{48} \mathrm{~N}_{3} \\
\mathrm{O}_{13}\end{array}$ & $\begin{array}{c}\mathrm{TbCu}_{4} \mathrm{I}_{4} \mathrm{C}_{46} \mathrm{H}_{48} \mathrm{~N}_{3} \\
\mathrm{O}_{13}\end{array}$ \\
\hline fw $\left(\mathrm{g} \mathrm{mol}^{-1}\right)$ & 1750.54 & 1752.75 & 1764.59 & 1771.55 \\
\hline $\begin{array}{l}\text { Crystal } \\
\text { system }\end{array}$ & triclinic & triclinic & triclinic & triclinic \\
\hline $\begin{array}{l}\text { space } \\
\text { group }\end{array}$ & $\mathrm{p}-1$ & $\mathrm{p}-1$ & $\mathrm{p}-1$ & $\mathrm{p}-1$ \\
\hline$a(\AA)$ & $9.776(2)$ & $9.758(2)$ & $9.6689(19)$ & $9.6050(19)$ \\
\hline$b(\AA)$ & $16.337(3)$ & $16.575(3)$ & $16.716(3)$ & $16.769(3)$ \\
\hline$c(\AA)$ & $21.534(4)$ & $21.452(4)$ & $21.277(4)$ & $21.155(4)$ \\
\hline$\alpha\left(^{\circ}\right)$ & $103.82(3)$ & $104.13(3)$ & $104.23(3)$ & $104.16(3)$ \\
\hline$\beta\left(^{\circ}\right)$ & $100.74(3)$ & $100.68(3)$ & $100.42(3)$ & $100.31(3)$ \\
\hline$\gamma\left({ }^{\circ}\right)$ & $95.87(3)$ & $96.53(3)$ & $96.86(3)$ & $97.16(3)$ \\
\hline$V\left(\AA^{3}\right)$ & $3242.3(11)$ & $3260.0(11)$ & $3229.5(11)$ & $3199.5(12)$ \\
\hline$Z$ & 2 & 2 & 2 & 2 \\
\hline$F(000)$ & 1666 & 1670 & 1680 & 1684 \\
\hline$\mu\left(\mathrm{mm}^{-1}\right)$ & 3.891 & 3.913 & 4.216 & 4.381 \\
\hline $\begin{array}{l}D_{\text {calcd }}(\mathrm{g} \\
\left.\mathbf{c m}^{-3}\right)\end{array}$ & 1.793 & 1.786 & 1.815 & 1.839 \\
\hline$R_{1}^{a}$ & 0.0612 & 0.0752 & 0.0758 & 0.0794 \\
\hline
\end{tabular}


Table S2. Selected bond lengths ( $\AA$ ) in Compound 1

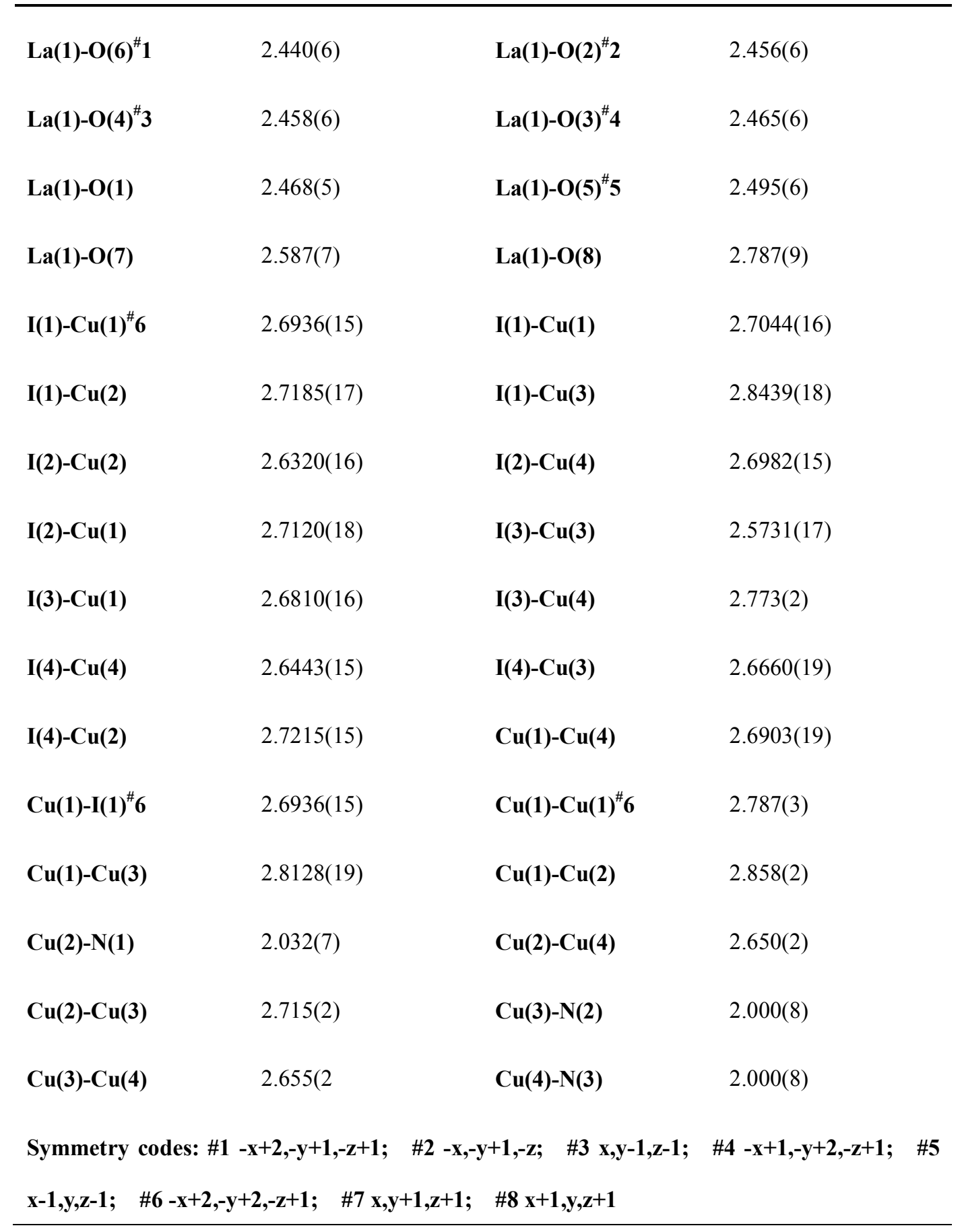


Table S3. Selected bond lengths ( $\AA$ ) in Compound 2

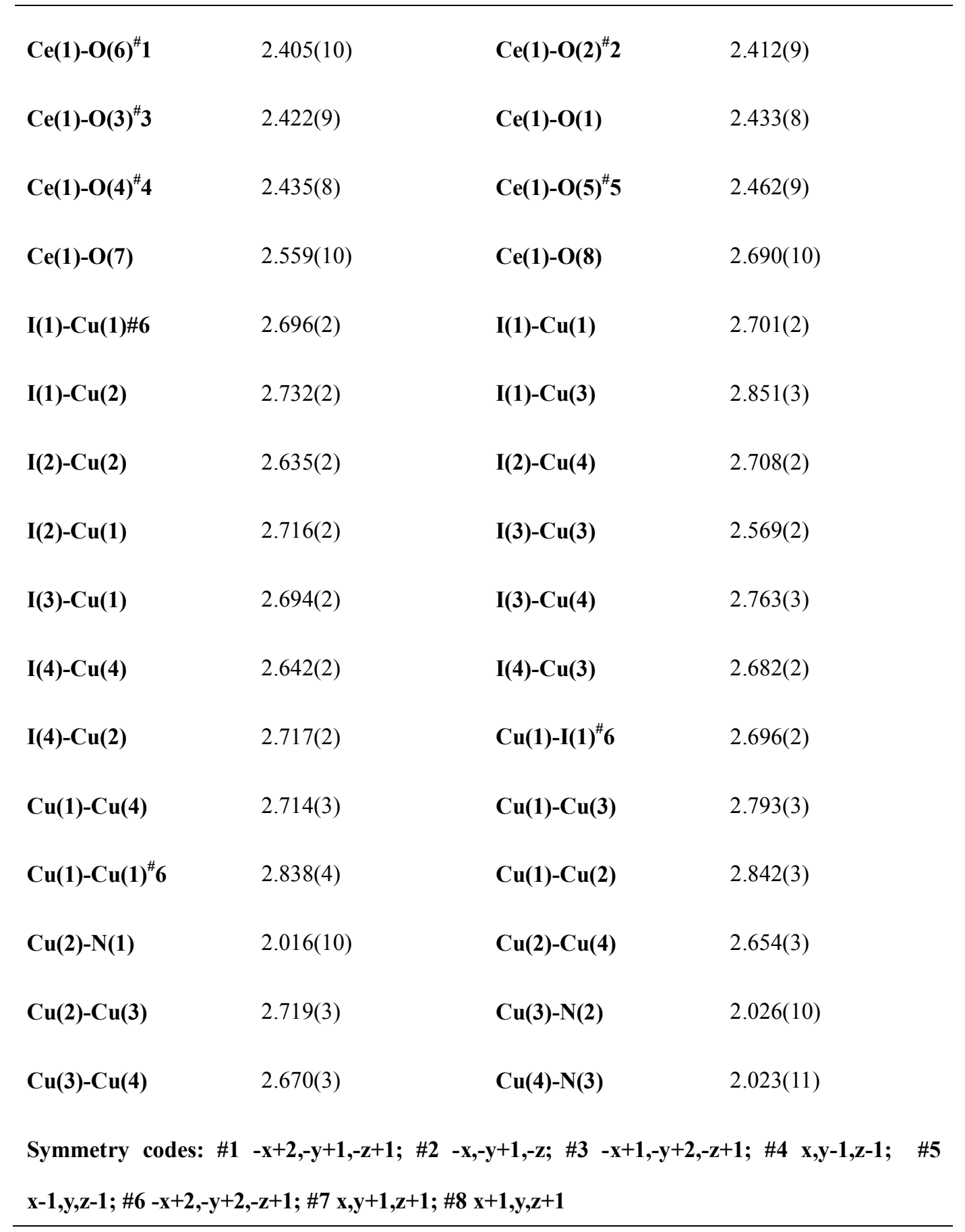


Table S4. Selected bond lengths ( $\AA$ ) in Compound 3

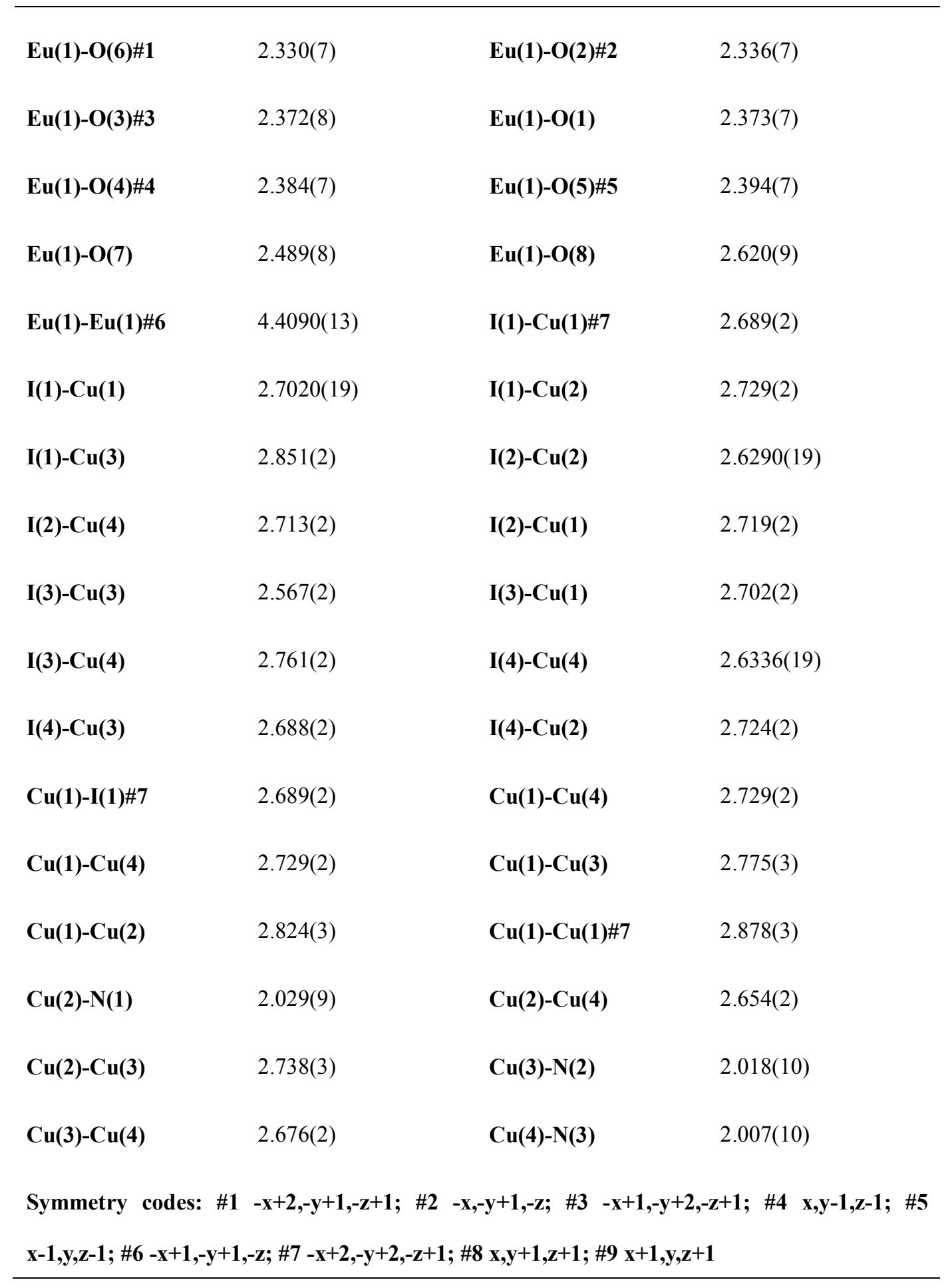


Table S5. Selected bond lengths $(\AA ̊)$ in Compound 4

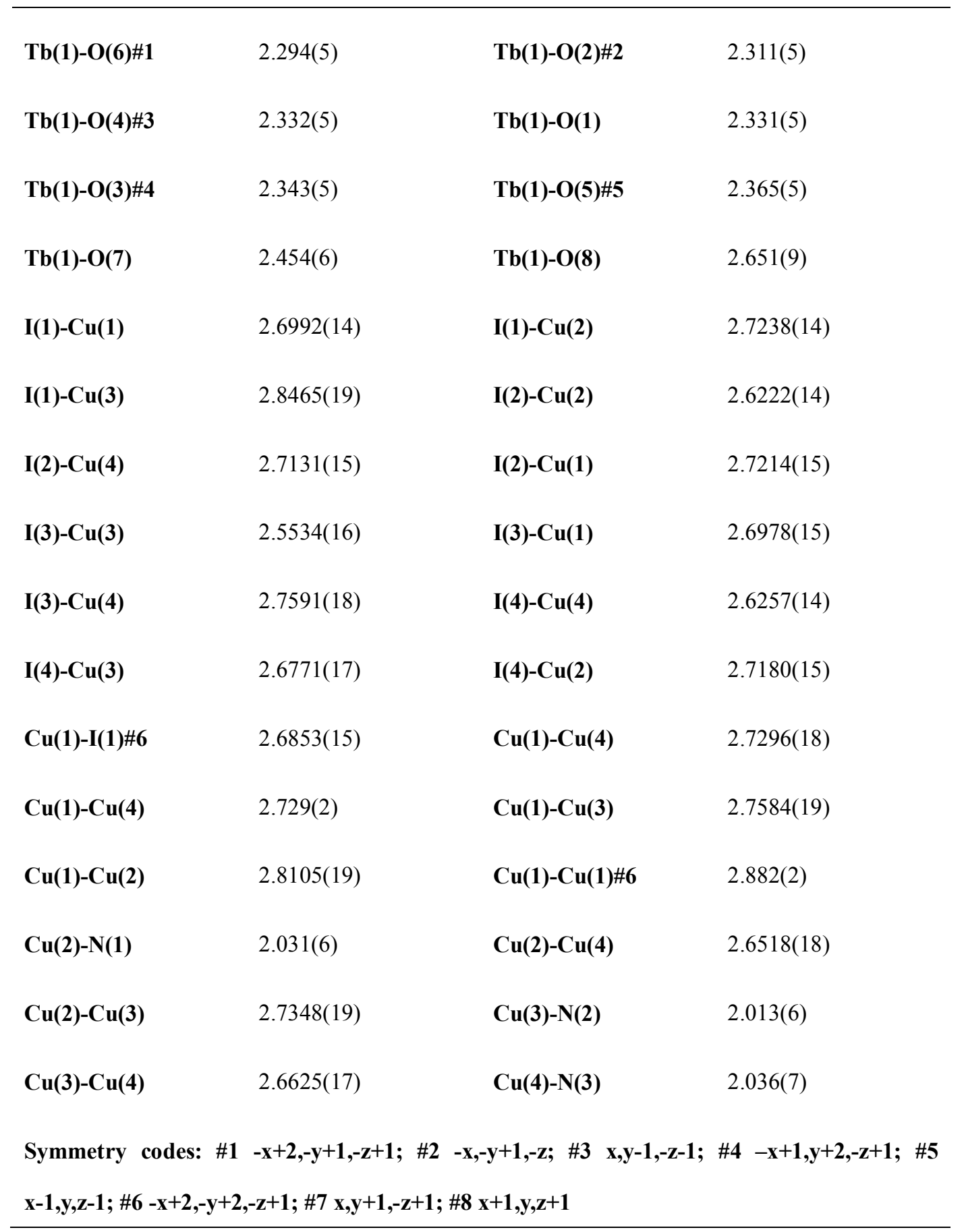




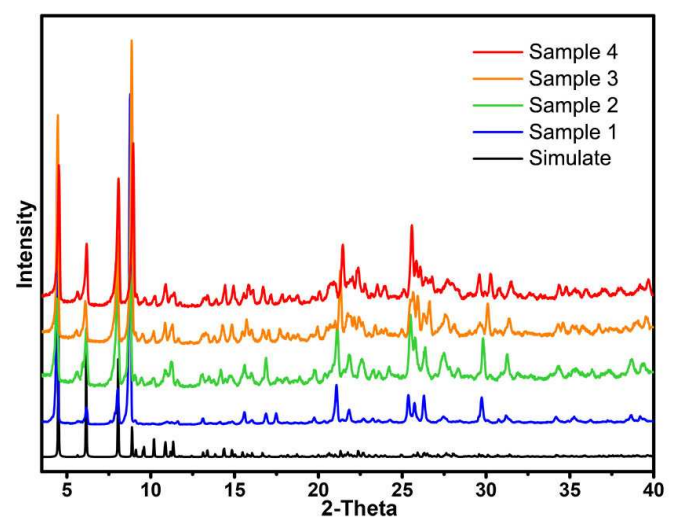

Figure S1. The XRD patterns of synthetic samples: Compound 1, 2, 3 and 4.

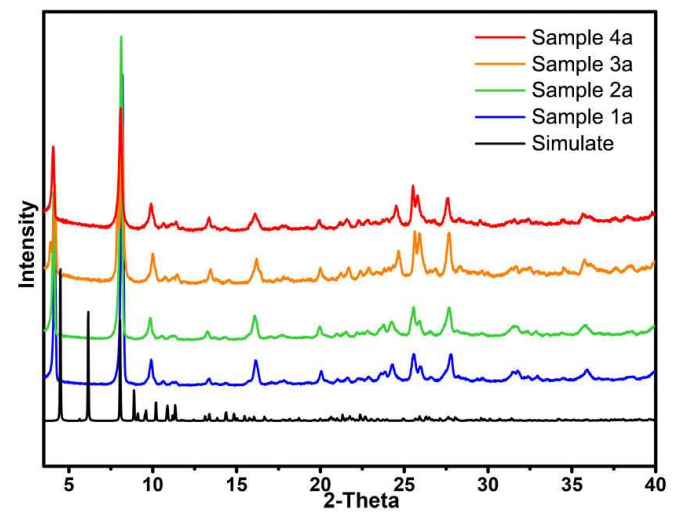

Figure S2.The XRD patterns of desolvated samples: sample 1a, 2a, 3a and 4a. 


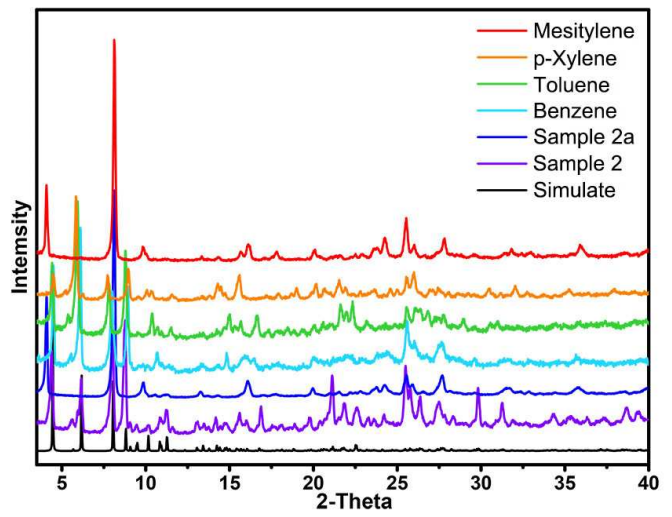

Figure S3. The XRD patterns of 2a $\supset$ guest (benzene, toluene, p-xylene and mesitylene). These results are well consistent with the XRD patterns of $4 \mathbf{a} \supset$ guest.

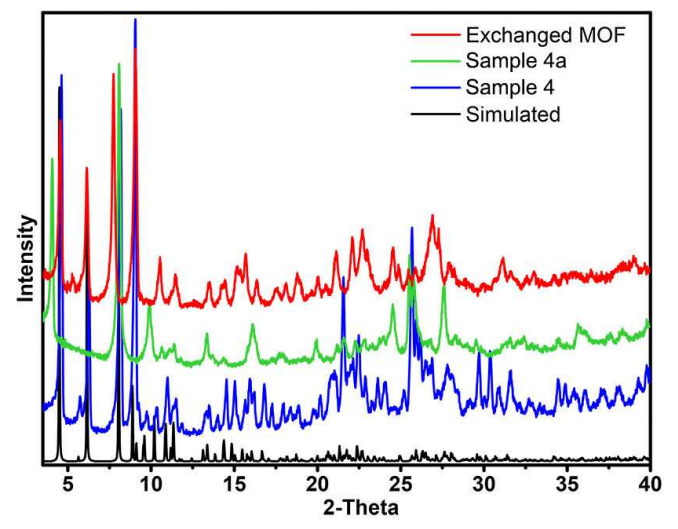

Figure S4. The XRD patterns of the solvent-exchanged sample. 


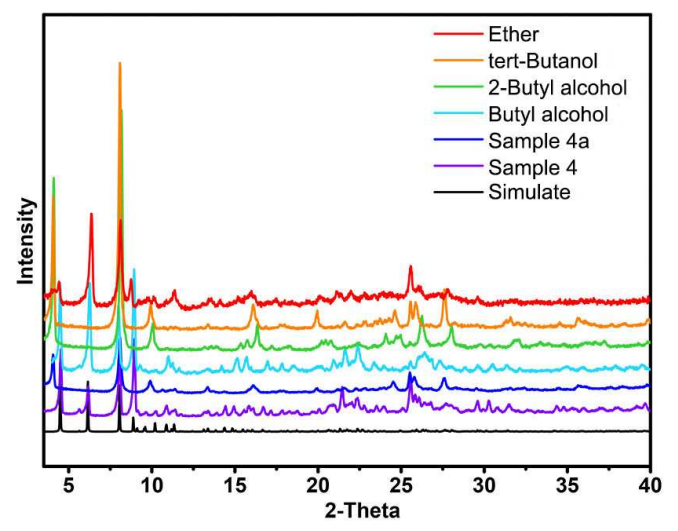

Figure S5. The XRD patterns of $\mathbf{4 a} \supset$ isomer (butyl alcohol, 2-butyl alcohol, tert-butanol and ether).

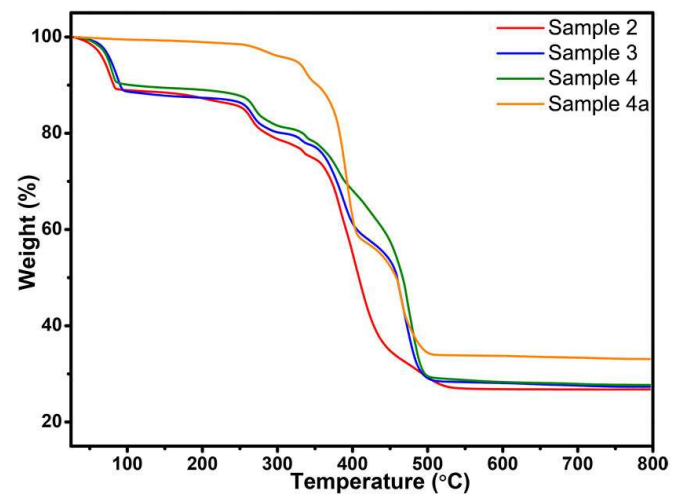

Figure S6. Thermogravimetric analysis curves of sample 2, 3, 4, and 4a. The results suggest the guest solvents have been remved. 


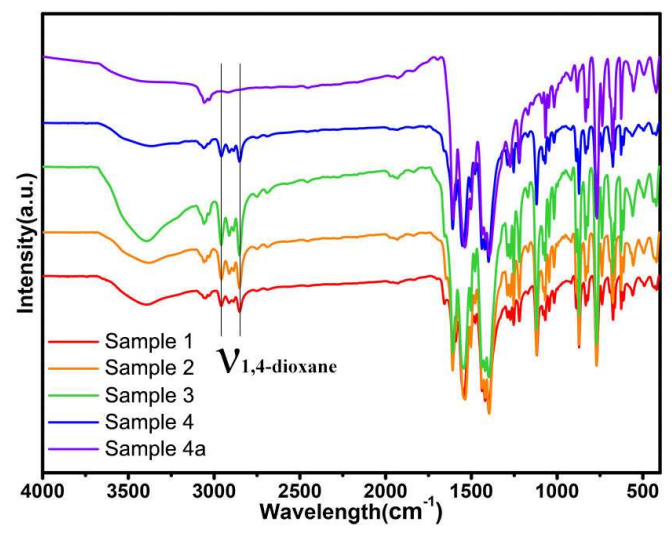

Figure S7. The IR spectra of sample 1, 2, 3, 4, and 4a. The characteristic IR absorption peaks of 1, 4-dioxane has disappeared in the IR spectra of $\mathbf{4 a}$. These results are well consistent with the TG analysis curves.

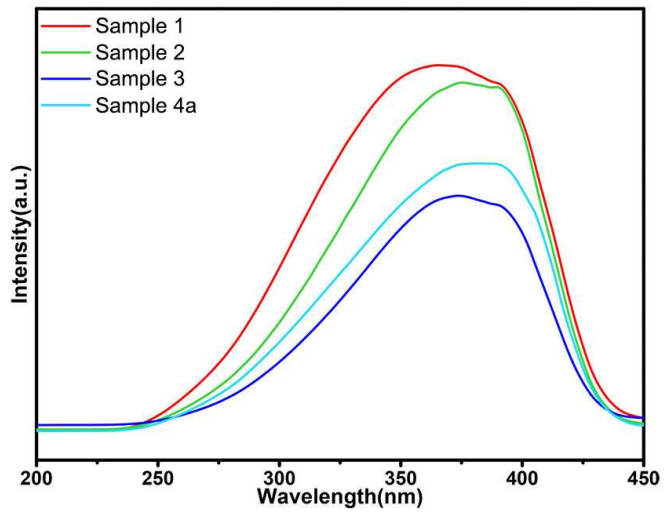

Figure S8. The excitation spectra of sample 1, 2, 3, and 4. 


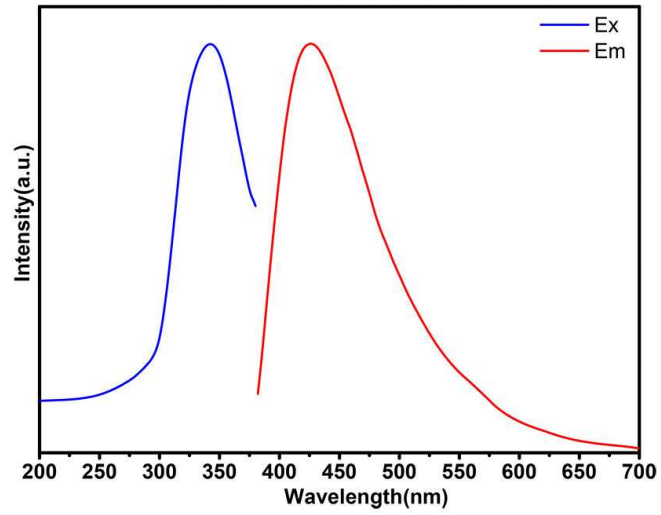

Figure S9. The luminescence spectra of the organic ligand (Hpba).

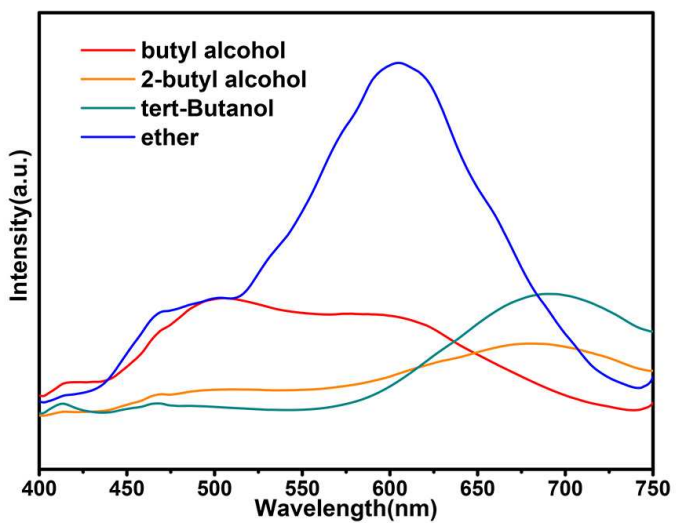

Figure S10. The luminescence spectra of 2a $\supset$ isomer (butyl alcohol, 2-butyl alcohol, tert-butanol and ether) after excitation at $380 \mathrm{~nm}$. 


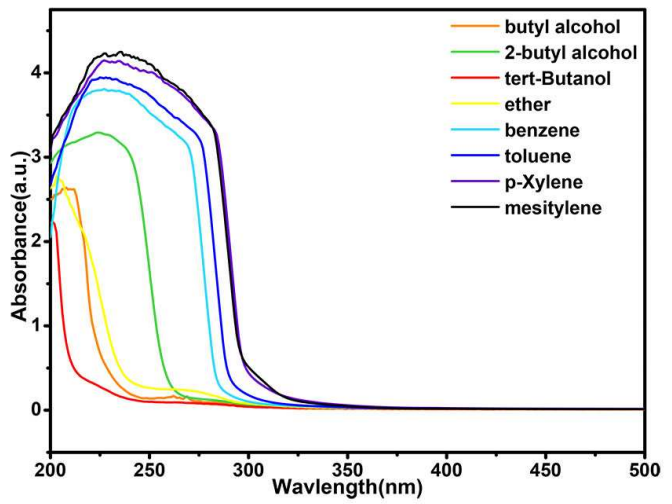

Figure S11. The UV-vis absorption spectra of solvent molecules: butyl alcohol, 2-butyl alcohol, tert-butanol, ether, benzene, toluene, p-xylene and mesitylene.

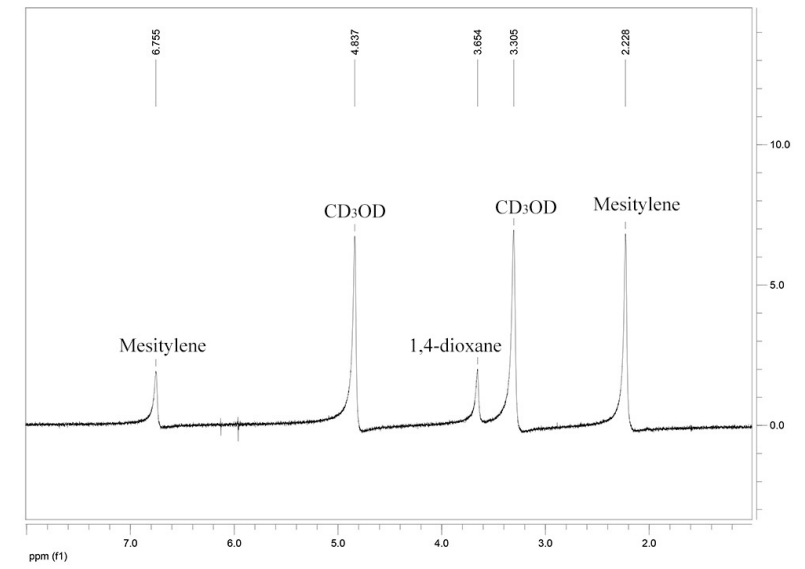

Figure S12. The ${ }^{1} \mathrm{H}$ NMR spectra in $\mathrm{CD}_{3} \mathrm{OD}$ of mesitylene-exchanged sample. The results suggest the mesitylene and 1, 4-dioxane coexist in the channel. 


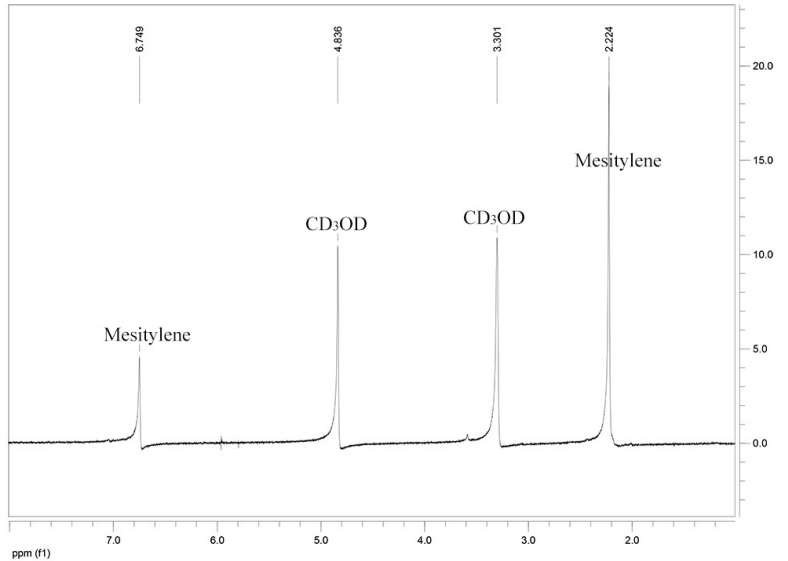

Figure S13. The ${ }^{1} \mathrm{H}$ NMR spectra in $\mathrm{CD}_{3} \mathrm{OD}$ of $4 \mathrm{a} \supset$ mesitylene. The result suggests the mesitylene has been successfully introduced into the framework.
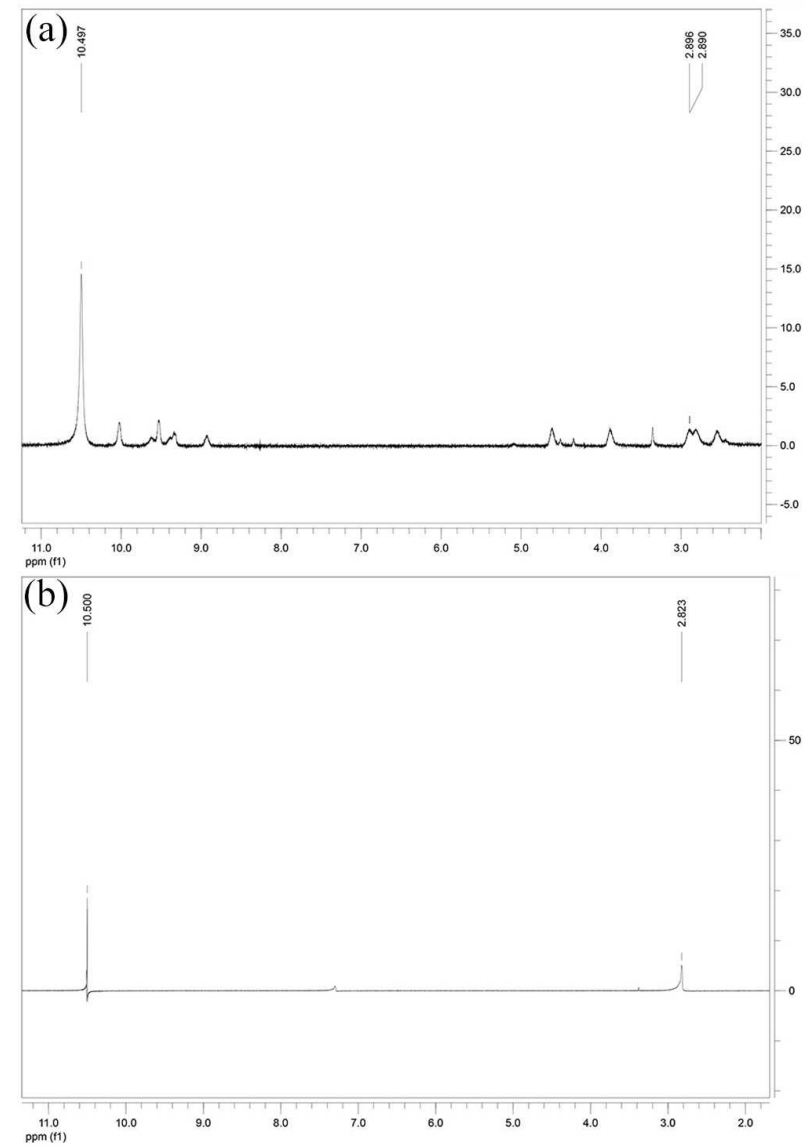

Figure S14. The ${ }^{1} \mathrm{H}$ NMR spectra of $\mathbf{4 a} \supset$ mesitylene (a) and mesitylene (b) after digested by deuterium chloride ( $\mathrm{DCl}$ ) solution in $\mathrm{D}_{2} \mathrm{O}$. The peaks which simultaneously appear in (a) and (b) also indicate mesitylene has been successfully introduced into the framework. 


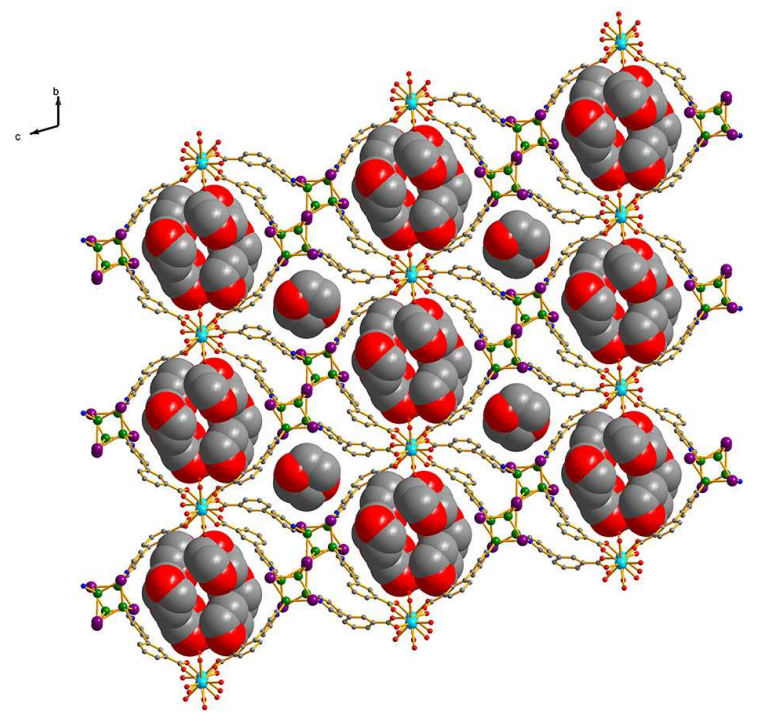

Figure S15. The 3D-stucture of Ln-MOFs and the channels fill with 1, 4-dioxane.

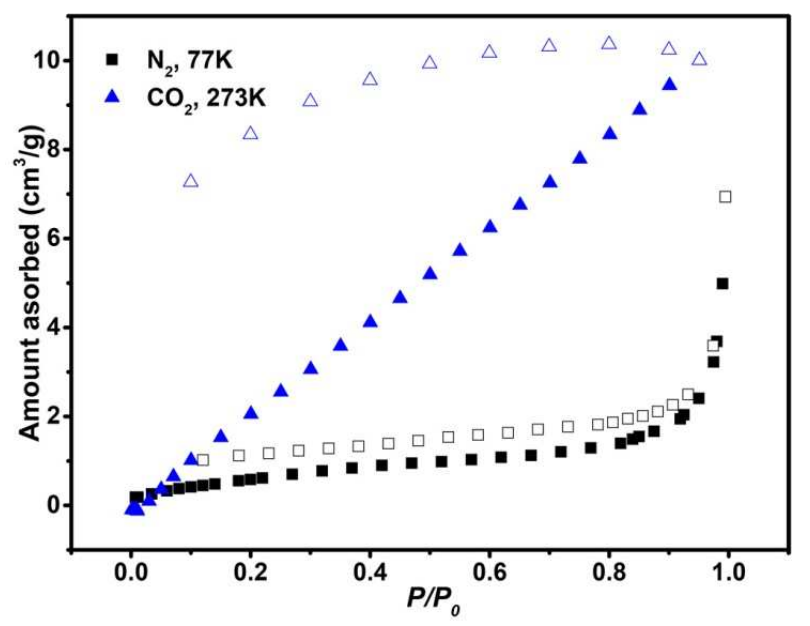

Figure S16. Adsorption (filled) and desorption (open) isotherms of N2 and $\mathrm{CO} 2$ gases under their condensable temperatures. The results suggest the activated sample has no obvious adsorption. 


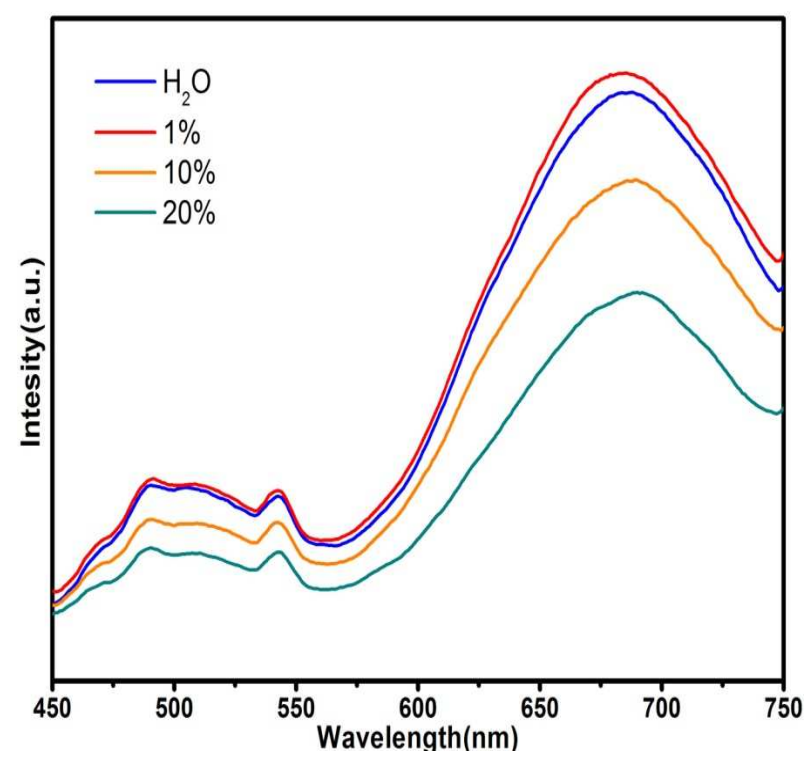

Figure S17. The luminescence spectra of the desolvent MOF (4a) after soaking in different concentrations of methanol aqueous solution. 